\title{
Patient reported outcomes to support drug development decision making
}

Annabel Nixon ${ }^{1}$, Diane Wild ${ }^{2}$, Willie Muehlhausen ${ }^{3}$

Chilli Consultancy, UK

Independent PRO and linguistic validation consultant, UK

ICON plc, UK

\section{INTRODUCTION}

Patient reported outcomes (PROs), a measurement based on a report that comes directly from the patient about the status of their health condition without amendment or interpretation of the patient's response by a clinician or anyone else [1], are playing an increasingly central role in drug development decision making. PRO instruments are available to measure signs and symptoms of disease, functioning (physical, cognitive, psychological, emotional), treatment satisfaction, activities of daily living and health-related quality of life (HRQL), amongst others. Valid and reliable PRO instruments are able to provide a standardized, quantifiable measure of treatment benefit, upon which the outcomes of interventions and treatment effect from the patient's perspective can be judged. In some instances, PROs provide the best evidence of a treatment's effectiveness, for example when evaluating treatments for pain, gastrointestinal and urological symptoms, or psychological well-being. This year the FDA approval of eluxadoline for the treatment of adults with irritable bowel syndrome with diarrhea (IBS-D) was based on a composite primary endpoint comprising patient reported daily worst abdominal pain score and a reduction in average daily stool consistency, with data being captured for both endpoints by patients in an electronic daily diary.

\section{APPLICATIONS OF PRO DATA}

PROs are used in clinical trials to measure the effect of an intervention on aspects of treatment relevant to patients. PRO data from clinical trials are submitted to regulatory agencies, such as the European Medicines Agency (EMA) and the US Food and Drug Administration (FDA), to support regulatory decision making. A review of 116 FDA ap- proved product labels 2006-2010, identified that $24 \%$ were granted PRO claims [2], with a comparison of FDA and EMA labelling showing that the EMA is more likely than the FDA to approve PRO claims [3]. The majority of PRO data that is captured does not make it onto the regulatory approved label, this is particularly noted in oncology [4] which is currently being actively addressed by industry and regulators [5]. PRO data, particularly HRQL data, provides essential contextualizing information in oncology so that the quality of improved survival can be understood from patient's perspective $[6,7]$.

PROs also provide important supporting evidence of treatment benefit that are of value to other stakeholders. Increasingly PROs are playing a central role in Health Technology Agency (HTA) decision making, particularly in the UK (National Institute for Clinical Excellence, NICE), France (Transparency Committee, TC) and Germany (Federal Joint Committee, GBA). The increasing role that economic evaluation plays in reimbursement decision making brings PROs to the forefront of HTA decision making as reimbursement agencies seek value propositions that go beyond traditional safety and clinical efficacy messages. Due to the rising costs of health care and the increased demand to demonstrate value for money, pharmaceutical companies are now charged with generating evidence on the patient's perspective of treatment. The patient's perspective is often expressed in the form of health utilities, a measure of satisfaction with a particular state of health, and PRO instruments (such as the EQ-5D5L, SF-6D) allow utilities to be derived from patients. Non-utility PRO data are increasingly valued by payors with recognition that they directly reflect the patient experience of a disease and its treatment, and contribute to a more holistic understanding of the poten-
Corresponding author Annabel Nixon anixon@chilli.global

\section{Disclosure}

The authors have no conflicts of interest to declare. The authors are also co-authors of a publication on PRO published by SEEd. 
tial value of a new product. Returning again to oncology, payors are increasingly interested in the quality as well as the duration of survival. Recent benefit assessments of the national HTA agency in Germany, the GBA (Federal Joint Committee), demonstrate how lack of such data can compromise pricing and reimbursement outcomes. In the assessment of crizotinib in non-small cell lung cancer (NSCLC) the manufacturer provided additional data during the hearing procedure with the GBA that allowed the evaluation of patient-reported symptoms and HRQL, the assessment was subsequently increased from 'no additional benefit proven' to a 'hint of significant additional benefit' versus chemotherapy in one patient subgroup.

PROs are also used in real world evidence or observational studies to capture the impact of a medical intervention on patients in real world settings, as well as in clinical practice to inform discussions between the physician and the patient. In the UK National Health Service (NHS) all patients having hip or knee replacements, varicose vein surgery, or groin hernia surgery are invited to fill in PROs, the data from the instruments are used to calculate the health gains after surgical treatment using pre- and post-operative surveys. National headline data are published every month with more granular data published every quarter. This data provides information on the effectiveness of care delivered to NHS funded patients from the perspective of the patients themselves, and complements existing information on the quality of NHS services.

PROs influence clinicians in prescribing decision making, and influence patient demands for treatments, particularly in the US where direct-to-consumer advertising is permitted.

\section{CHARACTERISTICS OF PRO INSTRUMENTS}

For PRO data to effectively inform decision making across all stakeholders, high standards of data capture and measurement are mandatory. PRO instruments are required to have proven validity, reliability, and ability to detect change (responsiveness). Great care has to be taken to ensure that PRO results are correctly interpreted, requiring published evidence of benchmarks used to evaluate PRO change scores in groups of patients (minimal important difference, MID) and individual patients (responder analysis). It is increasingly common for PROs to be used in multinational studies. For a PRO instrument to be considered valid to be used with a population who speak a different language than the language it was originally developed in, it needs to go through a specific translation process referred to as linguistic validation, which ensures that the translated PRO instrument has conceptual and semantic equivalence. The evaluation of technology and the rapid global penetration of mobile devices within patient populations has driven the adoption of electronic data capture of PROs. Technologies used to capture PRO data electronically include handheld devices, tablets, web-based systems, mobile web, interactive voice response systems (IVRS) and digital pen. There are substantial guidelines and best practice documents to support the use of electronic data capture systems in PRO assessment.

\section{CONCLUSIONS}

Patient reported outcomes can no longer be considered secondary to other outcome endpoints. Increasingly PRO data brings the patient voice to the forefront of decision making by multiple stakeholders: regulators, payors, health care providers, clinicians, prescribers, and increasingly health care consumers. It is almost certain that this is a trend set to continue alongside increasing calls for patient centeredness. Ultimately this leads to a need for PROs to receive the same level of attention as other endpoints in terms of selection, development, data collection, analysis and interpretation. The uniqueness of PRO data needs to be well understood in order to maximize decision outcomes.

These issues are explored in-depth in a publication co-authored by the editorial authors [Nixon A, Wild D, Muehlhausen W. Patient Reported Outcomes: an overview. Torino: SEEd, 2015] available online at: http://www.edizioniseed.it/ebooks/libri-patient-reported-outcomes.html and in the major online bookstores (i.e. www.amazon.com, https:// books.google.com/) 


\section{REFERENCES}

1. FDA (Food and Drug Administration). Guidance of Industry. Patient-reported outcome measures: use in medical product development to support labeling claims. December, 2009. Available at http:/www.fda.gov/downloads/ Drugs/Guidances/UCM193282.pdf

2. Gnanasakthy A, Mordin M, Clark M, et al. A review of patient-reported outcome labels in the United States: 2006 to 2010. Value in Health 2012: 15: 437-42; http://dx.doi.org/10.1016/j.jval.2011.11.032

3. DeMuro C, Clark M, Doward L, et al. Assessment of PRO label claims granted by the FDA as compared to the EMA (2006-2010). Value in Health 2013: 16: 1150-5; http://dx.doi.org/10.1016/j.jval.2013.08.2293

4. Tse J, Shingler S, Nixon A. The emerging role of patient-reported outcomes (PROs) in FDA hematology and oncology product labels. Value in Health 2015; 18: A210; http://dx.doi.org/10.1016/j.jval.2015.03.1216

5. Basch E, Geoghegan C, Coons SJ, et al. Patient-reported outcomes in cancer drug development. Perspectives from industry, the Food and Drug Administration, and the patient. JAMA Oncol 2015; 1:375-9; http://dx.doi.org/10.1001/ jamaoncol.2015.0530

6. Dimopoulos MA, Delforge M, Hajek R, et al. Lenalidomide, melphalan, and prednisone followed by lenalidomide maintenance improves health-related quality of life in newly diagnosed multiple myeloma patients aged 65 years or older: results of a randomized phase III trial. Haematologica 2013; 98: 784-8; http://dx.doi.org/10.3324/haematol.2012.074534

7. Minden MD, Dombret H, Seymour JF, et al. The effect of azacitidine on health-related quality of life (HRQL) in older patients with newly diagnosed acute myeloid leukemia: results from AZA-AML-001 trial. 20 $0^{\text {th }}$ Congress European Hematology Association. Vienna, 2015; Abstract P184 